\title{
High-performance coaxial wire-shaped supercapacitors using ionogel electrolyte toward sustainable energy system
}

\author{
Yongchao Liu ${ }^{1}$, Mugillan Narayanasamy ${ }^{1}$, Cheng Yang ${ }^{2}$, Minjie Shi ${ }^{1, a)}$, Wei Xie ${ }^{3}$, \\ Hanzhao $\mathrm{Wu}^{1}$, Chao Yan ${ }^{1, \mathrm{~b})}$, Hua Hou ${ }^{4}$, Zhanhu Guo ${ }^{5, \mathrm{c})}$ (D) \\ ${ }^{1}$ School of Materials Science and Engineering, Jiangsu University of Science and Technology, Zhenjiang 212003, China \\ ${ }^{2}$ State Key Laboratory for Metallic Matrix Composite Materials, School of Materials Science and Engineering, Shanghai Jiao Tong University, Shanghai \\ 200240, China \\ ${ }^{3}$ Key Laboratory of Lightweight and Reliability Technology for Engineering Vehicle, Education Department, Changsha University of Science \& \\ Technology, Changsha 45001, China \\ ${ }^{4}$ College of Materials Science and Engineering, North University of China, Taiyuan 030051, China \\ ${ }^{5}$ Integrated Composites Laboraotry (ICL), Department of Chemical and Biomolecular Engineering, University of Tennessee, Knoxville, Tennessee 37996, \\ USA; and Key Laboratory of Materials Processing and Mold (Zhengzhou University), Ministry of Education, National Engineering Research Center for \\ Advanced Polymer Processing Technology, Zhengzhou University, Zhengzhou 45001, China

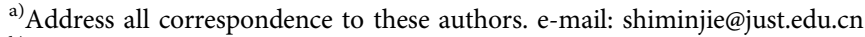 \\ b) e-mail: chaoyan@just.edu.cn \\ c) e-mail: zguo10@utk.edu
}

Received: 5 May 2019; accepted: 21 June 2019

Wire-shaped supercapacitors (WSSCs) hold great promise in portable and wearable electronics. Herein, a novel kind of high-performance coaxial WSSCs has been demonstrated and realized by scrolling porous carbon dodecahedrons/Al foil film electrode on vertical $\mathrm{FeOOH}$ nanosheets wrapping carbon fiber tows (FeOOH NSs/ CFTs) yarn electrode. Remarkably, ionogel is utilized as solid-state electrolyte and exhibits a high thermal/ electrochemical stability, which effectively ensures the great reliability and high operating voltage of coaxial WSSCs. Benefiting from the intriguing configuration, the coaxial WSSCs with superior flexibility act as efficient energy storage devices and exhibit low resistance, high volumetric energy density $\left(3.2 \mathrm{~mW} \mathrm{~h} / \mathrm{cm}^{3}\right)$, and strong durability ( $82 \%$ after 10,000 cycles). Importantly, the coaxial WSSCs can be effectively recharged by harvesting sustainable wind source and repeatedly supply power to the lamp without a decline of electrochemical performance. Considering the facile fabrication technology with an outstanding performance, this work has paved the way for the integration of sustainable energy harvesting and wearable energy storage units.

\section{Introduction}

Although there is a rapid growth of portable and wearable electronics $[1,2,3,4,5,6,7,8,9]$, a crucial challenge facing today is the development of lightweight, flexible, and highperforming energy storage devices $[10,11,12,13]$. Supercapacitors (SCs) showing inherent rapid charge/discharge characteristics alongside unlimited lifetime have been considered as promising energy storage devices to supply power for various electronic devices $[14,15,16,17,18,19,20]$. A recent development in wire-shaped SCs (WSSCs) shows great promise in portable and wearable electronics applications due to their less weight, tiny volume, and high flexibility [21, 22, 23, 24]. While several morphologies of WSSCs have been studied and reported, WSSCs in the shape of two twisted yarn electrodes have been explored extensively than the others. However, there are shortcomings with respect to acquiring efficient active area and large contact resistance between the two yarn electrodes in the fabricated WSSCs.

To circumvent these obstacles, WSSCs with coaxial structures can be designed and fabricated to provide the advantage of high active area for the electrode with much lower contact resistance leading to an efficient ionic diffusion and charge transport, in turn resulting in high electrochemical performance. $\mathrm{Xu}$ et al. [25] designed a coaxial WSSC by wrapping conducting carbon paper on $\mathrm{MnO}_{2}$-coated gold wire. The coaxial WSSCs can make full use of both the inner and outer 
active materials, which represent remarkable electrochemical behaviors, including low resistance, high specific capacitance, good rate, and cycle capability. To further enhance the mechanical stability, carbon fibers were applied to replace the metal wires as inner electrode. Le et al. [24] reported a coaxial fiber WSSC consisting of carbon fibers coated with carbon nanotubes as an inner electrode and carbon nanofiber film as an outer electrode. The fabricated WSSCs exhibited high flexibility and delivered a maximum energy density of $9.8 \mu \mathrm{W} \mathrm{h} / \mathrm{cm}^{2}$. Despite making progress, high energy density was not achieved in coaxial WSSCs based on aqueous gel electrolyte because of the narrow decomposition voltage of water $(<1.2 \mathrm{~V})$. Therefore, it still remains a challenging task to develop coaxial WSSC with a wide operating voltage and high energy density.

Since the energy density of SCs is proportional to the square of the operating voltage, the amount of energy stored in SCs depends essentially on the stability window of electrolytes. Ionic liquids (ILs) have been widely investigated as a kind of novel electrolytes for high-performance SCs because of the high operating voltages $(>2.5 \mathrm{~V})$, non-volatility, nonflammability, and high thermal/chemical stability [26, 27, 28, 29, 30]. Meanwhile, incorporating IL electrolyte into a polymer matrix can be used to produce a solid IL gel (also called ionogel) electrolyte. Similar to the IL electrolyte, ionogel served as the solid-state electrolyte, which can effectively improve the voltage range, thereby resulting in the high energy density of SCs [31, 32]. Nevertheless, to the best of our knowledge, there have been no reports on the construction of coaxial WSSCs using an ionogel electrolyte.

Herein, an ingenious strategy to fabricate coaxial WSSCs using an ionogel electrolyte has been reported. The WSSCs are realized by scrolling porous carbon dodecahedrons/Al foil (PCDs/ ALF) film electrode on vertical iron oxyhydroxide lepidocrocite (FeOOH) nanosheets wrapping carbon fiber tows (FeOOH NSs/ CFTs) yarn electrode. Benefiting from the intriguing configuration, the coaxial WSSCs with superior flexibility act as efficient energy storage devices exhibiting low resistances, ultrahigh energy densities, and a strong durability. As a result, the fabricated WSSCs with a high voltage of $3 \mathrm{~V}$ can be effectively recharged by harvesting sustainable wind energy and are capable of repeatedly lighting up a $1 \mathrm{~W}$ lamp for a long duration without a reduction in the electrochemical performance. Therefore, this work sheds light on the integration of sustainable energy harvesting and wearable energy storage devices.

\section{Results and discussion}

As represented in Fig. 1(a), the FeOOH NSs/CFTs yarn electrode has been synthesized by a simple one-step electrochemical deposition technique, which is directly used as the yarn electrode of WSSCs, and the digital photograph of
FeOOH NSs/CFTs yarn electrode is also shown in Fig. 1(a). Field emission scanning electron microscope (FESEM) images show the surface morphology of FeOOH NSs/CFTs yarn electrode. The FeOOH NSs/CFTs after growth $\mathrm{FeOOH}$ NS is separated from each other [Fig. 1(b)], retaining a large specific area of CFTs, which facilitates the rapid transfer of ions and electrons in the electrolyte. The enlarged highpower SEM images [Fig. 1(c)] show that the ultrathin FeOOH NSs are uniformly and vertically deposited with good conductivity and high flexibility of carbon fiber taws, and the vacant volume reserved between interleaved $\mathrm{FeOOH}$ NSs not only increases the surface area of the electrode but also provides a great quantity of highly electrochemically active sites $[33,34]$. In the X-ray diffraction (XRD) patterns [Fig. 1(d)] of CFTs and FeOOH NSs/CFTs, the broad diffraction peaks at $25^{\circ}$ correspond to the [002] diffraction of carbon, which is assigned to CFTs. The other diffraction peaks in $\mathrm{FeOOH} \mathrm{NSs/CFTs} \mathrm{are} \mathrm{attributed} \mathrm{to}$ the orthorhombic-type $\gamma$-FeOOH (JCPDS no. 08-0098). In addition, the $\mathrm{FeOOH}$ NSs/CFTs without other diffraction peaks indicate that uniform crystal was formed and no impurity sample was obtained [35, 36, 37]. The corresponding X-ray photoelectron spectroscopy (XPS) provides further information about the chemical states of FeOOH NSs/CFTs. There are two main peaks at binding energies of 711.3 and $724.8 \mathrm{eV}$ for $\mathrm{Fe} 2 p_{3 / 2}$ and $\mathrm{Fe} 2 p_{1 / 2}$, respectively, while with two shake-up satellite peaks at 719.5 and $732.1 \mathrm{eV}$, which are the typical characteristics of $\mathrm{Fe}^{3+}$ in $\mathrm{FeOOH}[38,39]$ [Fig. 1(e)]. Similarly, Fig. 1(f) shows the high-resolution O $2 p$ XPS spectrum of $\mathrm{FeOOH}$ NSs/CFTs, which could be fitted into the two main constituent peaks - namely, the Fe-O-Fe bond and $\mathrm{Fe}-\mathrm{O}-\mathrm{H}$ bond [37, 40, 41].

Figure $1(\mathrm{~g})$ shows the cyclic voltammetry $(\mathrm{CV})$ curves of the $\mathrm{FeOOH} \mathrm{NSs} / \mathrm{CFT}$ yarn electrode in $\mathrm{EMIMBF}_{4}$ IL electrolyte using three-electrode setup, which was tested over a wide range of -1.5 to $0.5 \mathrm{~V}$ at a scan rate of $10,20,50$, and $100 \mathrm{mV} / \mathrm{s}$. The $\mathrm{CV}$ profiles are characterized by redox peaks with the redox plateaus affirming the occurrence of a Faradaic process. Furthermore, FeOOH NSs/CFTs yarn electrode shows a rectangular shape at scan rates up to $100 \mathrm{mV} / \mathrm{s}$, indicating excellent electrochemical stability of the electrode. Figure 1(h) shows galvanostatic charge/discharge (GCD) curves of FeOOH NSs/CFTs yarn electrode from 1 to $8 \mathrm{~mA} / \mathrm{cm}^{2}$; the electrode delivers a high areal capacitance of $198 \mathrm{mF} / \mathrm{cm}^{2}$ at $1 \mathrm{~mA} / \mathrm{cm}^{2}$, which is mainly owing to the favorable ionic diffusion of $\mathrm{FeOOH} \mathrm{NSs} / \mathrm{CFT}$ yarn electrode in ionogel electrolyte. In addition, the FeOOH NSs/ CFTs yarn electrode exhibits excellent rate performance [Fig. 1(i)]; the capacitance measured at $10 \mathrm{~mA} / \mathrm{cm}^{2}$ still retains $75 \%$ of its value at $1 \mathrm{~mA} / \mathrm{cm}^{2}$.

A facile coating method for PCDs/ALF film electrode is shown in Fig. 2(a), and the detailed preparation process is 
(a)
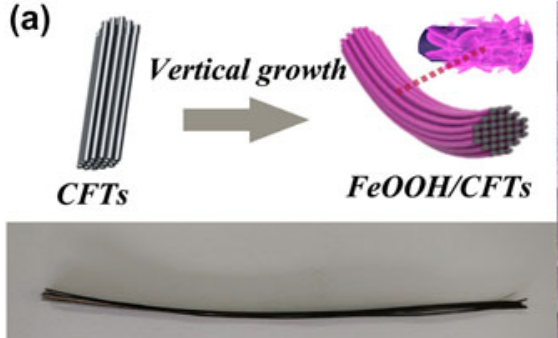

(d)

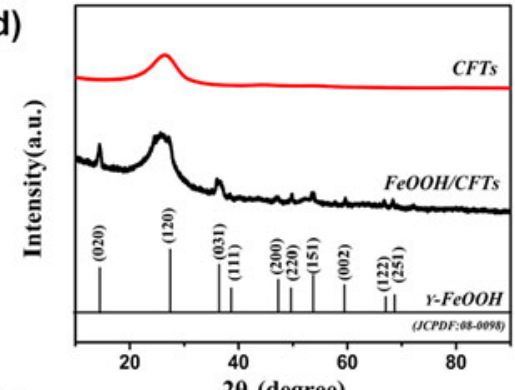

(g)

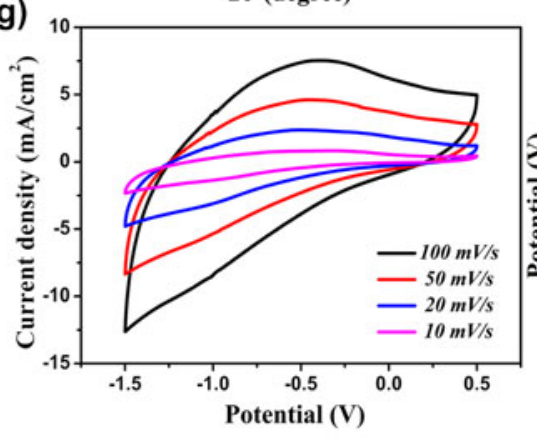


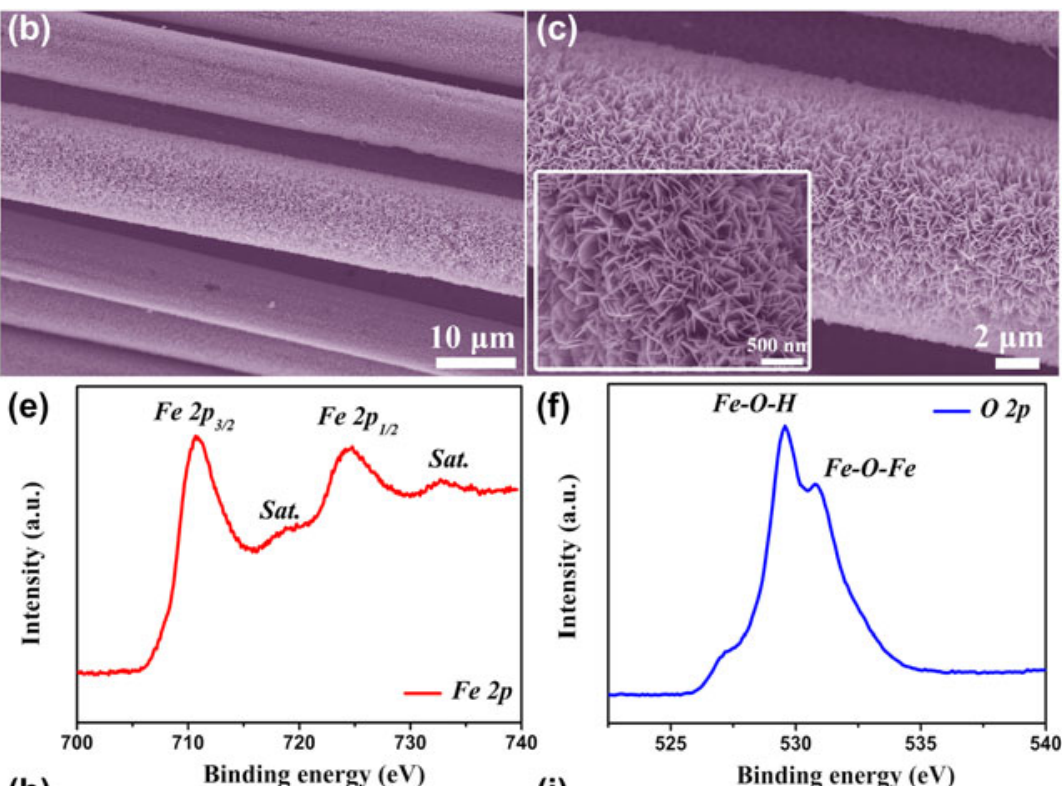

(h)

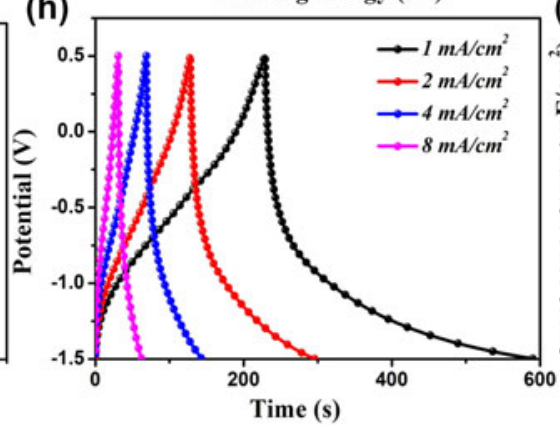

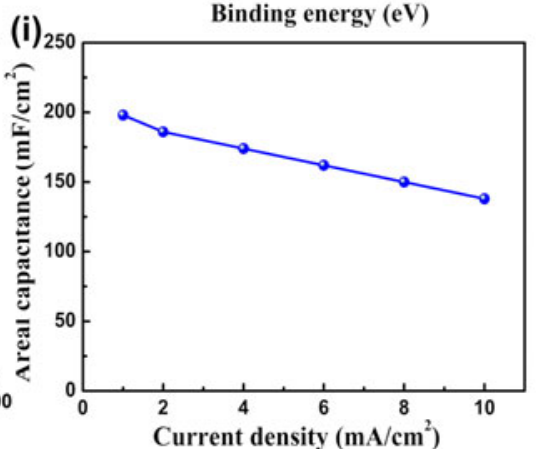

Figure 1: (a) Schematic and digital photograph of FeOOH NSs/CFTs yarn electrode. (b and c) SEM images of FeOOH NSs/CFTs. (d) XRD patterns of CFTs and $\mathrm{FeOOH}$ NSs/CFTs. High-resolution (e) Fe $2 p$ and (f) $02 p$ XPS spectrum of FeOOH NSs/CFTs. (g) CV curves of FeOOH NSs/CFTs yarn electrode at different scan rates. (h) GCD curves and (i) areal capacitance of FeOOH NSs/CFTs yarn electrode at different current densities.

described in "Experimental Section." FESEM image [Fig. 2(b)] reveals the microscopic morphology of PCDs/ALF film electrode. The magnified images of Fig. 2(c) further show that the uniform-sized polyhedral PCDs particles $(\approx 1 \mu \mathrm{m})$ are uniformly distributed on the surface of $\mathrm{Al}$ film with the action of conductive agent and binder, and form a stable $3 \mathrm{D}$ structure. The crystalline structure of PCDs was investigated by XRD [Fig. 2(d)], two broad peaks attributed to [002] and [101], confirming the amorphous structure of carbon [42, 43]. The pore structure of the PCDs was studied by the $\mathrm{N}_{2}$ adsorptiondesorption isotherms, as shown in Fig. 2(e). Obvious adsorption in the low-pressure region and the hysteresis loop in the medium- to high-pressure region indicate the presence of micropores and mesopores, respectively. The BrunauerEmmett-Teller specific surface area is $2060 \mathrm{~m}^{2} / \mathrm{g}$, with aplenty of pores $(<4 \mathrm{~nm})$. The micropores enable strong physical adsorption of ions in the electrolyte and the mesopores provide sufficient routes for ion migration [44, 45].

Figure 2(f) demonstrates the CV curves of PCDs/ALF film electrode and in three-electrode system with $\mathrm{EMIMBF}_{4}$ IL electrolyte at a potential window of $0-1.5 \mathrm{~V}$. The PCDs/ALF film electrode exhibits quasi-rectangular shape without obvious redox peaks at different scan rates, which reveal that the typical carbon-based EDLC-type charge storage behavior [46]. More importantly, the PCDs/ALF film electrode still exhibits a highly symmetrical CV curve at the scan rates up to $100 \mathrm{mV} / \mathrm{s}$, suggesting that the PCDs/ALF film electrode has a strong reversibility. The GCD curves of PCDs/ALF film electrode at different current densities from 1 to $8 \mathrm{~mA} / \mathrm{cm}^{2}$ [Fig. 2(g)] display typical capacitor triangle shapes, which are in good agreement with the highly symmetric CV profiles, and it is easily observed for non-Faradaic charge storage material [47]. The calculated specific capacity of the PCDs/ALF film electrode from GCD curves is $268 \mathrm{mF} / \mathrm{cm}^{2}$ at $1 \mathrm{~mA} / \mathrm{cm}^{2}$. The specific capacitances of PCDs/ALF film electrode at various current densities in Fig. 2(h) show the outstanding rate performance. Up to the high current density of $10 \mathrm{~mA} / \mathrm{cm}^{2}$, the PCDs/ALF film electrode delivers $196 \mathrm{mF} / \mathrm{cm}^{2}$.

Figure 3(a) depicts the assembly process of the $\mathrm{FeOOH}$ NSs/CFTs yarn electrode encapsulated by PCDs/ALF with 


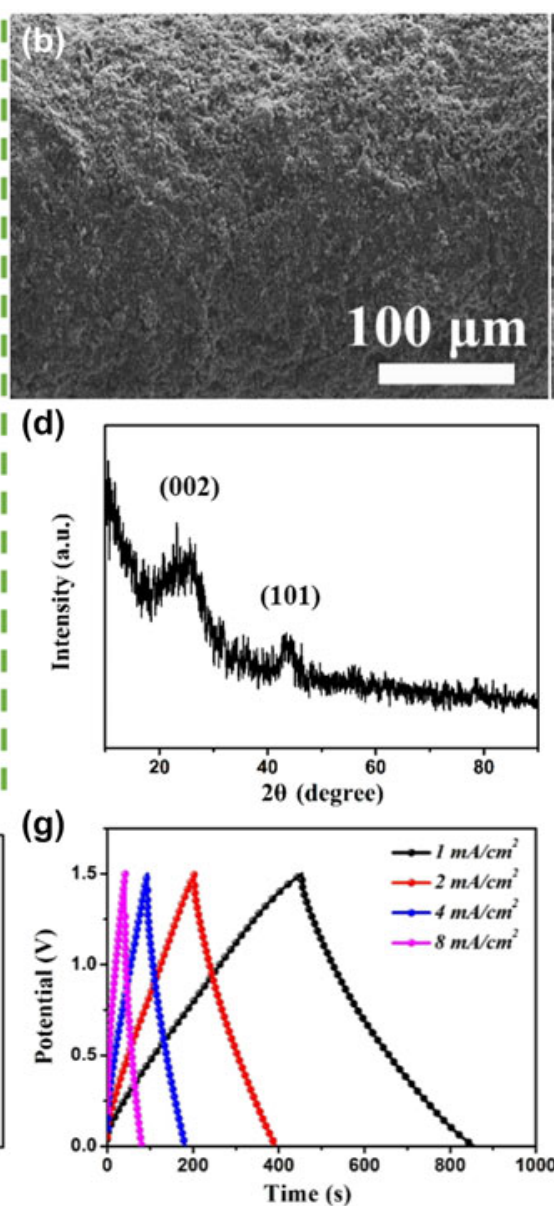

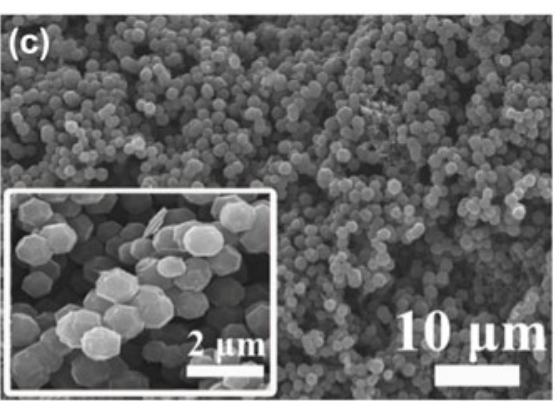

(e)

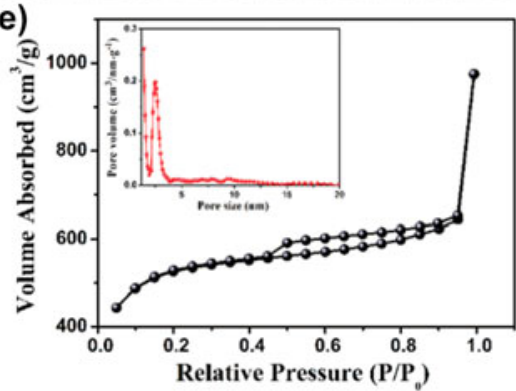

(h)

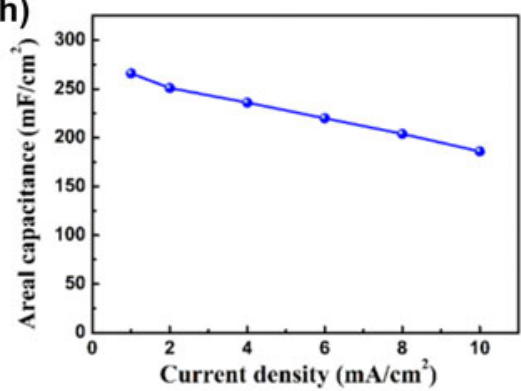

Figure 2: (a) Schematic diagram of the synthetic route of PCDs/ALF film electrode. (b and c) SEM images of PCDs/ALF film electrode with different magnifications. (d) XRD patterns and (e) $\mathrm{N}_{2}$ adsorption-desorption curve and pore size distribution (inset) of PCDs. (f) CV curves of PCDs/ALF film electrode at different scan rates. (g) GCD curves and (h) areal capacitance of PCDs/ALF film electrode at different current densities.

ionogel electrolyte as coaxial WSSCs, denoted as FeOOH NSs/ $\mathrm{CFTs} / / \mathrm{PCD} / \mathrm{ALF}$, in which PCDs/ALF is the positive electrode and $\mathrm{FeOOH} \mathrm{NSs} / \mathrm{CFT}$ s is the negative electrode material. The high specific surface area of FeOOH NSs/CFTs not only was fully utilized as the effective electrode area for coaxial WSSCs but also further ensures the mechanical stability, flexibility, and stretchability of coaxial WSSCs. The final fabricated coaxial WSSCs [Fig. 3(b)] was found to be $7 \mathrm{~cm}$ in length. The morphology of coaxial WSSCs was characterized by FESEM [Fig. 3(c)]. It is found that the FeOOH NSs/CFTs yarn electrode is completely and tightly encapsulated by PCDs/ ALF under the action of ionogel electrolyte. Figure 3(d) shows the CV curves of the WSSC device in the potential range of 0 $3 \mathrm{~V}$ at different scan rates. It is observed that the CV profiles are quasi-rectangular in shape with noticeable redox peaks, suggesting the existence of both Faradaic and non-Faradaic charge storage processes in the electrode. Moreover, the $\mathrm{CV}$ shape was well maintained even at high scan rate of $100 \mathrm{mV} / \mathrm{s}$, revealing fast charge and discharge properties of the WSSCs device. The reaction kinetics and charge transport properties of the WSSCs device were investigated by the electrochemical impedance spectroscopy (EIS) [Fig. 3(e)]. The typical Nyquist plot includes two regions: one is high-frequency region (inset), which is shown as a small semicircle indicating low charge transfer resistance $\left(R_{\mathrm{ct}}\right)$, and the other is low-frequency region, in which the impedance plot is quasi-vertical line indicating the excellent capacitive behavior of the WSSCs device [48, 49].

GCD curves of the as-fabricated coaxial WSSCs device at different current densities from 1 to $8 \mathrm{~mA} / \mathrm{cm}^{2}$ are presented in Fig. 4(a). Highly similar curves indicate excellent cycle stability of the device. By calculation, the device delivers excellent area capacitance of $72.5 \mathrm{mF} / \mathrm{cm}^{2}$ at the current density of $1 \mathrm{~mA} / \mathrm{cm}^{2}$. It is noted that the coaxial WSSCs device is stable without any appreciable degradation of electrolyte or redox of the electrodes up to a voltage of $3 \mathrm{~V}$. Moreover, the energy density of coaxial WSSCs is $68 \mu \mathrm{mW} \mathrm{h} /$ $\mathrm{cm}^{2}$ at the power density of $1.47 \mu \mathrm{W} / \mathrm{cm}^{2}$ and remains 41.1 $\mu \mathrm{mW} \mathrm{h} / \mathrm{cm}^{2}$ at $13.45 \mu \mathrm{W} / \mathrm{cm}^{2}$ [Fig. 4(b)], which is the among the best of various coaxial WSSCs reported in literature [24, $50,51,52,53,54,55]$. 

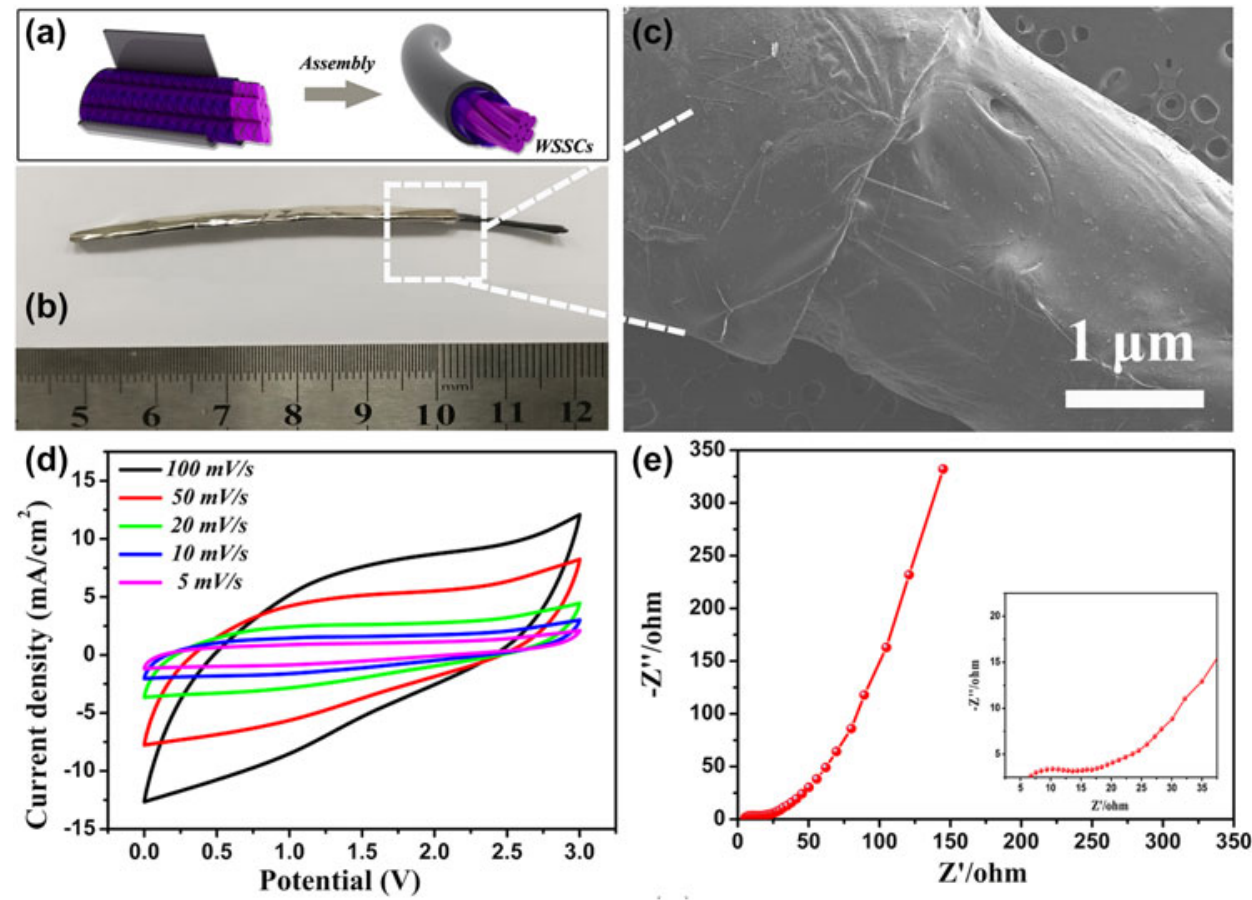

Figure 3: (a) Schematic diagram of assembly of FeOOH NSs/CFTs//PCDs/ALF coaxial WSSCs. (b) Digital photograph and (c) SEM image of coaxial WSSCs. (d) CV curves of fabricated coaxial WSSCs at different scan rates. (e) The Nyquist plots of coaxial WSSCs.
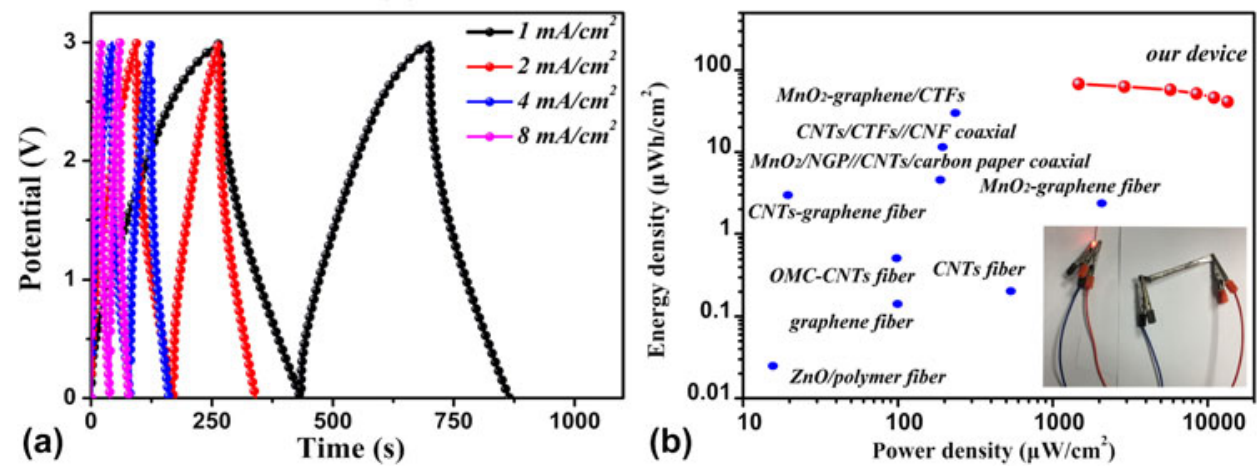

Figure 4: (a) GCD curves of coaxial WSSCs at different current densities. (b) The Ragone plots displaying energy and power densities of fabricated coaxial WSSCs device in comparison with other SCS; inset shows WSSCS device can lighten up a red LED indicator.

To further investigate the robustness and mechanical strength of the fabricated device and to study the device electrochemical performances, the as-fabricated coaxial WSSCs device was twisted into different angles [Fig. 5(a)]. The device is found to have good flexibility and mechanical stability, indicating that the coaxial WSSCs device possesses the desired functional characteristics. The flexible device showed excellent capacitive stability when the device was bent in various bending angles [Fig. 5(b)]. The device displays almost $100 \%$ overlap of $\mathrm{CV}$ curves at the bending angles of $0^{\circ}, 50^{\circ}, 100^{\circ}$, and $180^{\circ}$, demonstrating good electrochemical stability of the device. The durability test of the device was carried out by performing 10,000 cycles under straight and curved conditions; the device was charged and discharged at a current density of $200 \mathrm{~mA} /$ $\mathrm{cm}^{2}$, which is shown in Fig. 5(c). The device retains $87 \%$ straight and $82 \%$ bending of its initial capacitance after the cycling tests, demonstrating its good stability with long cycle life.

The development of miniaturized storage device has a great significance for the use of renewable clean energy, especially for easy access to natural energy. Previous literature reports integrating wind-driven generator (WDG) with SCs, due to the fast charging-discharging capability and long cycle life of SCs $[56,57,58]$. For a real application, the system was built using the universal availability of harvesting sustainable wind energy with the speed of $22-28 \mathrm{~km} / \mathrm{h}$ [Fig. $6(\mathrm{a})$ ]. The real- 




(a)

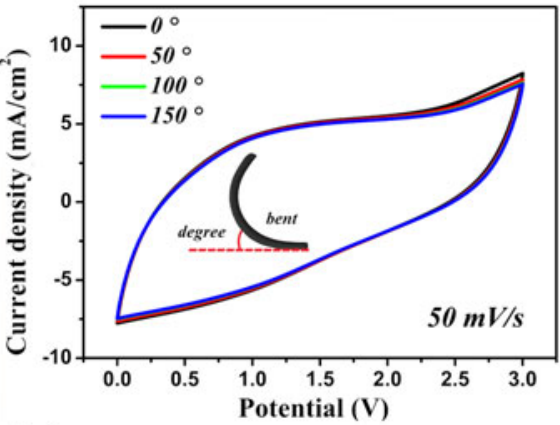

(b)

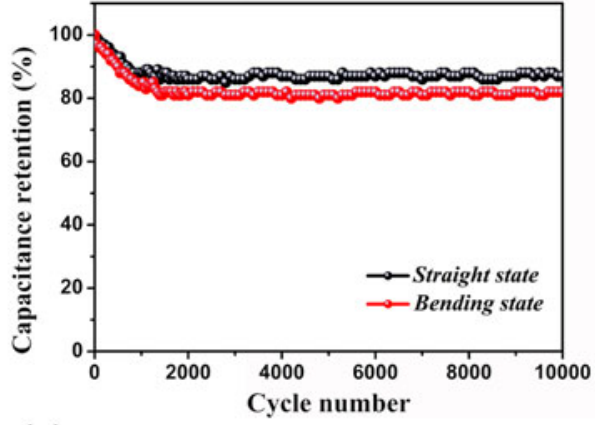

(c)

Figure 5: (a) Stability test of coaxial WSSCs device under different bending conditions. (b) CV curves of the device under different mechanical bending angles at a scan rate of $50 \mathrm{mV} / \mathrm{s}$. (c) Capacitance retention test of the device in the straight and bending conditions.

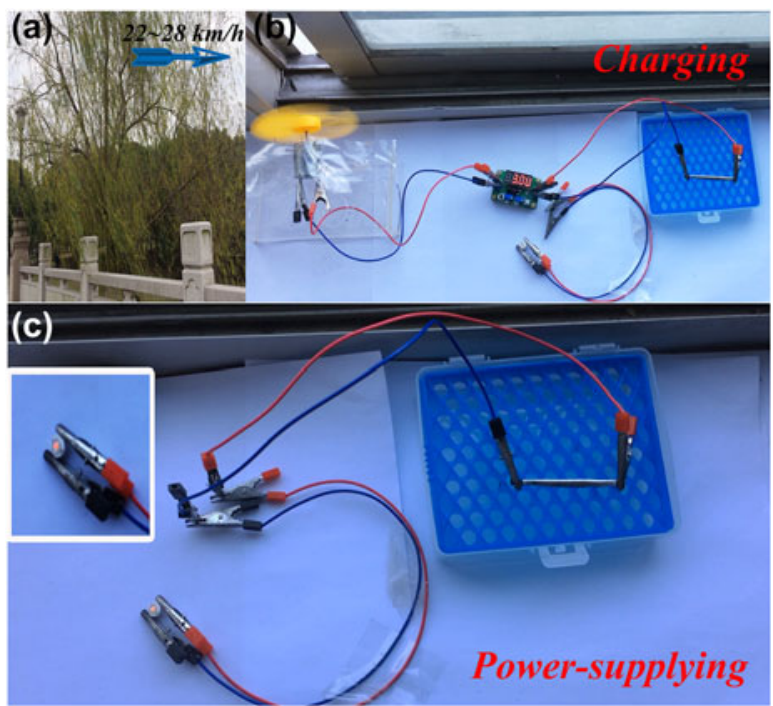

Figure 6: Proof of concept in wind energy applications. ( $a$ and b) Real-time photograph of the charging process of the WSSCs by harvesting sustainable wind energy. (c) Digital image shows the WSSCs as efficient wearable energy storage devices after charging can lighten up a red LED indicator.

time photograph [Fig. 6(b)] depicts the charging process of the fabricated coaxial WSSCs device that connected to a miniature WDG, followed by an electronic regulator. Depending on the circuit configuration, wind energy from the WDG powers the electronic regulator, supplying a constant output current to the coaxial WSSCs and storing the harvested energy. Figure 6(c) demonstrates the performance of the fabricated WSSCs device soon after charging with wind energy, while the inset clearly shows the lighting of the commercial red indicator (1 W/1.6 V). Thus, the fabricated WSSCs device was able to charge itself from the wind power and deliver the stored energy for glowing the light emitting diode (LED). The outcome of the present study clearly suggests the possible potential utilization of the textile structures for commercial usage in the future. This method can be utilized in industrial scale to fabricate a device to store and supply energy to portable electronic appliances during a power outage in the grid lines as this device can be charged using wind power.

\section{Conclusion}

In summary, a kind of high-performance coaxial WSSCs has been demonstrated and is realized by encapsulating PCDs/ALF film electrode on $\mathrm{FeOOH}$ NSs/CFTs yarn electrode. Remarkably, the vertically oriented $\mathrm{FeOOH} \mathrm{NSs}$ and cross-linked electroactive network on CFTs are much favorable for the ionic diffusion and charge transport in between PCDs/ALF film. Furthermore, ionogel utilized as solid-state electrolyte exhibits high thermal/electrochemical stability, which effectively ensures the great reliability and high operating voltage of coaxial WSSCs. Compared with other energy conversion and storage units $[59,60,61,62,63,64,65,66]$, coaxial WSSCs not only show superior flexibility and wearability but also exhibit low resistance, high volumetric energy density (3.2 $\mathrm{mW} \mathrm{h} / \mathrm{cm}^{3}$ ), and strong durability ( $82 \%$ after 10,000 cycles). More importantly, the coaxial WSSCs device with high voltage of $3 \mathrm{~V}$ can be effectively recharged by harvesting sustainable wind source and repeatedly supply power to the lamp without a decline of electrochemical performance.

\section{Experimental section}

\section{Preparation of FeOOH NSs/CFTs yarn electrode}

All the reagents were of analytical grade and were used without further purification. Pristine CFTs with a diameter of $1.6 \mathrm{~mm}$ were placed into concentrated nitric acid after cleaning with acetone, ethanol, and deionized water for several times and dried at room temperature. The treated CFTs, as the working electrode, was immersed into the solution mix containing $0.01 \mathrm{M} \mathrm{Fe}\left(\mathrm{NH}_{4}\right)_{2}\left(\mathrm{SO}_{4}\right)_{2} \cdot 6 \mathrm{H}_{2} \mathrm{O}$ and $0.04 \mathrm{M} \mathrm{CH}{ }_{3} \mathrm{COOK}$ in the solvent mixture of water and ethanol in 9:1 ratio by volume. A platinum foil and an $\mathrm{Ag} /$ $\mathrm{AgCl}$ electrode were set as counter and the reference 
electrodes, respectively. Vertical FeOOH NSs were electrodeposited on CFTs under a constant potential of $0.65 \mathrm{~V}$ at $70{ }^{\circ} \mathrm{C}$ for $1 \mathrm{~h}$. After that, the $\mathrm{FeOOH}$ NSs/CFTs yarn electrode was washed with deionized water to remove residual solvents and dried at $60{ }^{\circ} \mathrm{C}$ in a vacuum oven.

\section{Preparation of PCDs/ALF film electrode}

First, PCDs were derived from a zeolitic imidazole metalorganic framework (ZIF-8). Zinc acetate $(48 \mathrm{mM})$ was dissolved in $200 \mathrm{~mL}$ methanol solution containing polyvinyl pyrrolidone $(0.4 \mathrm{mM})$. The mixture was then placed in a refrigerator $\left(\sim 4{ }^{\circ} \mathrm{C}\right)$ for $1 \mathrm{~h}$. Meanwhile, $200 \mathrm{~mL}$ of 2 methylimidazole $(160 \mathrm{mM})$ methanol solution was cooled in the same condition. Sequentially, the two solutions were mixed and incubated at room temperature overnight. The ZIF- 8 was received under vacuum drying after centrifugation and washing, which was then calcined at $900{ }^{\circ} \mathrm{C}$ for $5 \mathrm{~h}$ under $\mathrm{Ar}$ atmosphere to obtain PCD powder. After that, the mixture of resultant PCDs (85 wt\%), Super P (5 wt\%), and polytetrafluoroethylene (PTFE) (10 wt\%) was uniformly coated onto the surface of flexible ALF, followed by a drying-solidifying process in the oven at $60{ }^{\circ} \mathrm{C}$ to form PCDs/ALF film electrode.

\section{Assembling of coaxial WSSCs}

Ionogel electrolyte was prepared with $0.5 \mathrm{~g}$ poly(vinylidene fluoride-hexafluoropropylene) (PVDF-HFP), $2 \mathrm{~g} \mathrm{EMIMBF}_{4} \mathrm{IL}$, and $4 \mathrm{~mL}$ of acetone solution. The FeOOH NSs/CFTs yarn electrode (cut into $5 \mathrm{~cm}$ ) was lightly twisted to form a round bundle for use as the inner electrode of coaxial WSSCs. Meanwhile, PCDs/ALF film electrode was cut into $5 \mathrm{~cm} \times$ $0.5 \mathrm{~cm}$ pieces. The well-prepared yarn and film electrodes were both immersed into the viscous electrolyte solution, with end part ( $\sim 1 \mathrm{~cm}$ in length) kept above the solution. After being taken out, two electrodes were completely encapsulated in the ionogel electrolyte. At this moment, PCDs/ALF film as the outer electrode was tightly surrounded onto the inner electrode of FeOOH NSs/CFTs yarn, in which the ionogel electrolyte acted as a glue to attach the two electrodes well. Finally, coaxial WSSCs were obtained after the ionogel electrolyte solidified at room temperature.

\section{Fabrication of wind-charging power system}

The miniature WDG was purchased Zongqing Gabosun Technology Co., Ltd., Shijiazhuang, China, with a rotor containing four blades (total diameter $\approx 10 \mathrm{~cm}$ ). A miniature WDG, an electronic regulator, and a coaxial WSSC were connected with each other according to the circuit schematic, in which the WDG converted wind power into electricity, electronic regulator with the adjusted voltage of $3 \mathrm{~V}$ was used to supply constant output current to a coaxial WSSCs, and the WSSCs store the harvested energy. Simulative wind source is from draught fan. To demonstrate the feasibility of the WSSCs charged by wind power, a commercial red lamp $(1 \mathrm{~W} / 1.6 \mathrm{~V})$ was used.

\section{Characterization}

FESEM (FEI Sirion 200, Japan) and transmission electron microscopy (TEM; JEM-2010F, Japan) were carried out to characterize the morphology of the samples. XRD patterns were characterized on a powder XRD system with $\mathrm{Cu} \mathrm{K}_{\alpha}$ radiation, and XPS measurements were performed on a Kratos AXIS Ultra DLD spectrometer (U.K.) with Al $\mathrm{K}_{\alpha}$ X-ray source. All the electrochemical measurements were carried out using a VMP3 multifunctional electrochemical analysis instrument (Bio-Logic, France). Electrochemical behaviors were investigated by $\mathrm{CV}, \mathrm{GCD}$, and EIS methods using a VMP3 multifunctional electrochemical analysis instrument (Bio-Logic, France). The CV and GCD tests were carried out at various scan rates and current densities. The EIS plots were performed in the frequency ranging from $0.05 \mathrm{~Hz}$ to $100 \mathrm{kHz}$ with $5 \mathrm{mV}$ AC amplitude. The specific capacitance, energy, and power density of coaxial WSSCs were calculated from the galvanostatic discharge curve according to the two-electrode systematic calculation method:

$$
\begin{gathered}
C_{\mathrm{v}}=I \Delta t / V \Delta U, \\
E_{\mathrm{v}}=C_{\mathrm{v}}(\Delta U)^{2} / 7.2, \\
P_{\mathrm{v}}=3600 \cdot E_{\mathrm{v}} / \Delta t,
\end{gathered}
$$

where $C_{\mathrm{v}}\left(\mathrm{mF} / \mathrm{cm}^{2}\right)$ is the areal specific capacitance, $E_{\mathrm{v}}(\mu \mathrm{W}$ $\left.\mathrm{h} / \mathrm{cm}^{3}\right)$ is the areal energy density, $P_{\mathrm{v}}\left(\mu \mathrm{W} / \mathrm{cm}^{3}\right)$ is the areal power density, $I$ is the discharge current, $\Delta t$ is the discharge time, $\Delta U$ is the voltage variation during the discharge process, and $V\left(\mathrm{~cm}^{2}\right)$ is the total area of fabricated devices. Additionally, the electrochemical performance and areal specific capacitance of single FeOOH NSs/CFTs yarn or PCDs/ALF film electrode were measured according to the three-electrode systematic method in $\mathrm{EMIMBF}_{4}$ IL electrolyte.

\section{Acknowledgments}

Yongchao Liu and Mugilan Narayanasamy contributed equally to this work. We greatly acknowledge the funding for this project through the National Natural Science Foundations of China (No. 51873083), the Opening Project of State Key Laboratory of Polymer Materials Engineering (Sichuan University) (No. sklpme2018-4-27), the Six Talent Peaks Project in Jiangsu Province (No. 2015-XCL-028), and the Postgraduate Research \& Practice Innovation Program of Jiangsu Province (KYCX17-1831 and SJCX18-0759). 


\section{Supplementary material}

To view supplementary material for this article, please visit https://doi.org/10.1557/jmr.2019.234.

\section{References}

1. H. Liu, Q. Li, S. Zhang, R. Yin, X. Liu, Y. He, K. Dai, C. Shan, J. Guo, C. Liu, C. Shen, X. Wang, N. Wang, Z. Wang, R. Wei, and Z. Guo: Electrically conductive polymer composites for smart flexible strain sensors: A critical review. J. Mater. Chem. C 6 , 12121-12141 (2018).

2. S. Zhang, H. Liu, S. Yang, X. Shi, D. Zhang, C. Shan, L. Mi, C. Liu, C. Shen, and Z. Guo: Ultrasensitive and highly compressible piezoresistive sensor based on polyurethane sponge coated with a cracked cellulose nanofibril/silver nanowire layer. ACS Appl. Mater. Interfaces 11, 10922-10932 (2019).

3. Q. Li, H. Liu, S. Zhang, D. Zhang, X. Liu, Y. He, L. Mi, J. Zhang, C. Liu, C. Shen, and Z. Guo: Superhydrophobic electrically conductive paper for ultrasensitive strain sensor with excellent anticorrosion and self-cleaning property. ACS Appl. Mater. Interfaces 11, 21904-21914 (2019).

4. C. Shen, X. Liu, H. Cao, Y. Zhou, J. Liu, J. Tang, X. Guo, H. Huang, and X. Chen: Brain-like navigation scheme based on MEMS-INS and place recognition. Appl. Sci. 9, 1708 (2019).

5. Y.W.D. Jiang, B. Li, C. Sun, Z. Wu, H. Yan, L. Xing, S. Qi, Y. Li, H. Liu, W. Wei, X. Wang, T. Ding, and Z. Guo: Flexible sandwich structural strain sensor based on silver nanowires decorated self-healing substrate. Macromol. Mater. Eng. 304, 1900074 (2019).

6. H. Gu, H. Zhang, C. Ma, H. Sun, C. Liu, K. Dai, J. Zhang, R. Wei, T. Ding, and Z. Guo: Smart strain sensing organicinorganic hybrid hydrogels with nano barium ferrite as the crosslinker. J. Mater. Chem. C 7, 2353-2360 (2019).

7. H. Liu, Y. Li, K. Dai, G. Zheng, C. Liu, C. Shen, X. Yan, J. Guo, and Z. Guo: Electrically conductive thermoplastic elastomer nanocomposites at ultralow graphene loading levels for strain sensor applications. J. Mater. Chem. C 4, 157-166 (2016).

8. Y. Lu, M.C. Biswas, Z.H. Guo, J.W. Jeon, and E.K. Wujcik: Recent developments in bio-monitoring via advanced polymer nanocomposite-based wearable strain sensors. Biosens. Bioelectron. 123, 167-177 (2019)

9. H. Wei, H. Wang, Y. Xia, D. Cui, Y. Shi, M. Dong, C. Liu, T. Ding, J. Zhang, Y. Ma, N. Wang, Z. Wang, Y. Sun, R. Wei, and Z. Guo: An overview of lead-free piezoelectric materials and devices. J. Mater. Chem. C 6, 12446-12467 (2018).

10. W. Liu, M.S. Song, B. Kong, and Y. Cui: Flexible and stretchable energy storage: Recent advances and future perspectives. $A d v$ Mater. 29, 1603436 (2017).

11. C. Yang, X. Ji, X. Fan, T. Gao, L. Suo, F. Wang, W. Sun, J. Chen, L. Chen, F. Han, L. Miao, K. Xu, K. Gerasopoulos, and C. Wang:
Flexible aqueous Li-ion battery with high energy and power densities. Adv. Mater. 29, 1701972 (2017).

12. J. Zhang, Z. Zhang, Y. Jiao, H. Yang, Y. Li, J. Zhang, and P. Gao: The graphene/lanthanum oxide nanocomposites as electrode materials of supercapacitors. J. Power Sources 419, 99-105 (2019).

13. K. Sun, R.H. Fan, X.H. Zhang, Z.D. Zhang, Z.C. Shi, N. Wang, P.T. Xie, Z.Y. Wang, G.H. Fan, H. Liu, C.T. Liu, T.X. Li, C. Yan, and Z.H. Guo: An overview of metamaterials and their achievements in wireless power transfer. J. Mater. Chem. C 6, 2925-2943 (2018).

14. Y. Jiao, J. Zhang, S. Liu, Y. Liang, S. Li, H. Zhou, and J. Zhang: The graphene oxide ionic solvent-free nanofluids and their battery performances. Sci. Adv. Mater. 10, 1706-1713 (2018).

15. Y.C. Liu, M.J. Shi, C. Yan, Q.Q. Zhuo, H.Z. Wu, L. Wang, H. Liu, and Z.H. Guo: Inspired cheese-like biomass-derived carbon with plentiful heteroatoms for high performance energy storage. J. Mater. Sci. 30, 6583-6592 (2019).

16. K. Le, Z. Wang, F. Wang, Q. Wang, Q. Shao, V. Murugadoss, S. Wu, W. Liu, J. Liu, Q. Gao, and Z. Guo: Sandwich-like NiCo layered double hydroxide/reduced graphene oxide nanocomposite cathodes for high energy density asymmetric supercapacitors. Dalton Trans. 48, 5193-5202 (2019).

17. Y. Ma, C. Hou, H. Zhang, Q. Zhang, H. Liu, S. Wu, and Z. Guo: Three-dimensional core-shell $\mathrm{Fe}_{3} \mathrm{O}_{4} /$ polyaniline coaxial heterogeneous nanonets: Preparation and high performance supercapacitor electrodes. Electrochim. Acta 315, 114-123 (2019).

18. W. Du, X. Wang, J. Zhan, X. Sun, L. Kang, F. Jiang, X. Zhang, Q. Shao, M. Dong, H. Liu, V. Murugadoss, and Z. Guo: Biological cell template synthesis of nitrogen-doped porous hollow carbon spheres $/ \mathrm{MnO}_{2}$ composites for high-performance asymmetric supercapacitors. Electrochim. Acta 296, 907-915 (2019).

19. B. Kirubasankar, V. Murugadoss, J. Lin, T. Ding, M. Dong, H. Liu, J. Zhang, T. Li, N. Wang, Z. Guo, and S. Angaiah: In situ grown nickel selenide on graphene nanohybrid electrodes for high energy density asymmetric supercapacitors. Nanoscale 10, 20414-20425 (2018).

20. W. Deng, T. Kang, H. Liu, J. Zhang, N. Wang, N. Lu, Y. Ma, A. Umar, and Z. Guo: Potassium hydroxide activated and nitrogen doped graphene with enhanced supercapacitive behavior. Sci. Adv. Mater. 10, 937-949 (2018).

21. X. Dong, Z. Guo, Y. Song, M. Hou, J. Wang, Y. Wang, and Y. Xia: Flexible and wire-shaped micro-supercapacitor based on $\mathrm{Ni}(\mathrm{OH})_{2}$-nanowire and ordered mesoporous carbon electrodes. Adv. Funct. Mater. 24, 3405 (2014)

22. D. Yu, Q. Qian, L. Wei, W. Jiang, K. Goh, J. Wei, J. Zhang, and Y. Chen: Emergence of fiber supercapacitors. Chem. Soc. Rev. 44, 647 (2015)

23. L. Kou, T. Huang, B. Zheng, Y. Han, X. Zhao, K. Gopalsamy, H. Sun, and C. Gao: Coaxial wet-spun yarn supercapacitors for high-energy density and safe wearable electronics. Nat. Commun. 5, 3754 (2014) 
24. V.T. Le, H. Kim, A. Ghosh, J. Kim, J.M. Chang, Q.A. Vu, D.T. Pham, J-H. Lee, S-W. Kim, and Y.H. Lee: Coaxial fiber supercapacitor using all-carbon material electrodes. ACS Nano 7, 5940-5947 (2013).

25. H. Xu, X. Hu, Y. Sun, H. Yang, X. Liu, and Y. Huang: Flexible fiber-shaped supercapacitors based on hierarchically nanostructured composite electrodes. Nano Res. 8, 1148-1158 (2015).

26. R. Thangavel, A.G. Kannan, R. Ponraj, V. Thangavel, D-W. Kim, and Y-S. Lee: High-energy green supercapacitor driven by ionic liquid electrolytes as an ultra-high stable nextgeneration energy storage device. J. Power Sources 383, 102-109 (2018).

27. J. Chapman Varela, K. Sankar, A. Hino, X. Lin, W. Chang,

D. Coker, and M. Grinstaff: Piperidinium ionic liquids as electrolyte solvents for sustained high temperature supercapacitor operation. Chem. Commun. 54, 5590 (2018).

28. S. Shahzad, A. Shah, E. Kowsari, F.J. Iftikhar, A. Nawab, B. Piro, M.S. Akhter, U.A. Rana, and Y. Zou: Ionic liquids as environmentally benign electrolytes for high-performance supercapacitors. Global Challeng 3, 1800023 (2019).

29. J. Zhang, P. Li, Z. Zhang, X. Wang, J. Tang, H. Liu, Q. Shao,

T. Ding, A. Umar, and Z. Guo: Solvent-free graphene liquids: Promising candidates for lubricants without the base oil. J. Colloid Interface Sci. 542, 159-167 (2019).

30. J. Zhang, S. Liu, C. Yan, X. Wang, L. Wang, Y. Yu, and S. Li: Abrasion properties of self-suspended hairy titanium dioxide nanomaterials. Appl. Nanosci. 7, 691-700 (2017).

31. X. Zhang, M. Kar, T.C. Mendes, Y. Wu, and D.R. MacFarlane: Supported ionic liquid gel membrane electrolytes for flexible supercapacitors. Adv. Energy Mater. 8, 1702702 (2018).

32. L. Feng, K. Wang, X. Zhang, X. Sun, C. Li, X. Ge, and Y. Ma: Flexible solid-state supercapacitors with enhanced performance from hierarchically graphene nanocomposite electrodes and ionic liquid incorporated gel polymer electrolyte. Adv. Funct. Mater. 28, 1704463 (2018).

33. J-Q. Xie, Y-Q. Ji, J-H. Kang, J-L. Sheng, D-S. Mao, X-Z. Fu, R. Sun, and C-P. Wong: In situ growth of $\mathrm{Cu}(\mathrm{OH})_{2} @ \mathrm{FeOOH}$ nanotube arrays on catalytically deposited $\mathrm{Cu}$ current collector patterns for high-performance flexible in-plane micro-sized energy storage devices. Energy Environ. Sci. 12, 194-205 (2019).

34. J. Li, Y. Wang, W. Xu, Y. Wang, B. Zhang, S. Luo, X. Zhou, C. Zhang, X. Gu, and C. Hu: Porous $\mathrm{Fe}_{2} \mathrm{O}_{3}$ nanospheres anchored on activated carbon cloth for high-performance symmetric supercapacitors. Nano Energy 57, 379-387 (2019).

35. L. Yang, M. Shi, J. Jiang, Y. Liu, C. Yan, H. Liu, and Z. Guo: Heterogeneous interface induced formation of balsam pear-like PPy for high performance supercapacitors. Mater. Lett. 244, 27-30 (2019).

36. Z. Qu, M. Shi, H. Wu, Y. Liu, J. Jiang, and C. Yan: An efficient binder-free electrode with multiple carbonized channels wrapped by $\mathrm{NiCo}_{2} \mathrm{O}_{4}$ nanosheets for high-performance capacitive energy storage. J. Power Sources 410-411, 179-187 (2019).
37. F. Xin, Y. Jia, J. Sun, L. Dang, Z. Liu, and Z. Lei: Enhancing the capacitive performance of carbonized wood by growing $\mathrm{FeOOH}$ nanosheets and poly(3,4-ethylenedioxythiophene) coating. ACS Appl. Mater. Interfaces 10, 32192-32200 (2018).

38. B. Liu, Y. Wang, H.Q. Peng, R. Yang, Z. Jiang, X. Zhou, C.S. Lee, H. Zhao, and W. Zhang: Iron vacancies induced bifunctionality in ultrathin feroxyhyte nanosheets for overall water splitting. Adv. Mater. 30, 1803144 (2018).

39. H. Luo, K. Tao, and Y. Gong: K-doped $\mathrm{FeOOH} / \mathrm{Fe}_{3} \mathrm{O}_{4}$ nanoparticles grown on a stainless steel substrate with superior and increasing specific capacity. Dalton Trans. 48, 2491-2504 (2019).

40. X. Han, C. Yu, J. Yang, X. Song, C. Zhao, S. Li, Y. Zhang,

H. Huang, Z. Liu, H. Huang, X. Tan, and J. Qiu:

Electrochemically driven coordination tuning of $\mathrm{FeOOH}$ integrated on carbon fiber paper for enhanced oxygen evolution. Small 15, 1901015 (2019).

41. J. Liu, M. Zheng, X. Shi, H. Zeng, and H. Xia: Amorphous $\mathrm{FeOOH}$ quantum dots assembled mesoporous film anchored on graphene nanosheets with superior electrochemical performance for supercapacitors. Adv. Funct. Mater. 26, 919-930 (2016).

42. F. Zheng, Y. Yang, and Q. Chen: High lithium anodic performance of highly nitrogen-doped porous carbon prepared from a metal-organic framework. Nat. Commun. 5, 5261 (2014).

43. Y.P. Li, C.H. Yang, F.H. Zheng, X. Ou, Q.C. Pan, Y.Z. Liu, and G. Wang: High pyridine N-doped porous carbon derived from metal-organic frameworks for boosting potassium-ion storage. J. Mater. Chem. A 6, 17959-17966 (2018).

44. K. Chen, Z. Sun, R. Fang, Y. Shi, H-M. Cheng, and F. Li: Metalorganic frameworks (MOFs)-derived nitrogen-doped porous carbon anchored on graphene with multifunctional effects for lithium-sulfur batteries. Adv. Funct. Mater. 28, 1707592 (2018).

45. L.F. Chen, Y. Lu, L. Yu, and X.W. Lou: Designed formation of hollow particle-based nitrogen-doped carbon nanofibers for high-performance supercapacitors. Energy Environ. Sci. 10, 1777-1783 (2017).

46. L. Chen, R. Lin, and C. Yan: Nitrogen-doped double-layer graphite supported $\mathrm{CuCo}_{2} \mathrm{~S}_{4}$ electrode for high-performance asymmetric supercapacitors. Mater. Lett. 235, 6-10 (2019).

47. P. Sun, R. Lin, Z. Wang, M. Qiu, Z. Chai, B. Zhang, H. Meng, S. Tan, C. Zhao, and W. Mai: Rational design of carbon shell endows TiN@C nanotube based fiber supercapacitors with significantly enhanced mechanical stability and electrochemical performance. Nano Energy 31, 432-440 (2017).

48. S-Y. Lu, M. Jin, Y. Zhang, Y-B. Niu, J-C. Gao, and C.M. Li Chemically exfoliating biomass into a graphene-like porous active carbon with rational pore structure, good conductivity, and large surface area for high-performance supercapacitors. Adv. Energy Mater. 8, 1702545 (2017).

49. X. Wu, Z. Han, X. Zheng, S. Yao, X. Yang, and T. Zhai: Coreshell structured $\mathrm{Co}_{3} \mathrm{O}_{4} @ \mathrm{NiCo}_{2} \mathrm{O}_{4}$ electrodes grown on flexible carbon fibers with superior electrochemical properties. Nano Energy 31, 410-417 (2017). 
50. Z. Zhang, F. Xiao, and S. Wang: Hierarchically structured $\mathrm{MnO}_{2} /$ graphene/carbon fiber and porous graphene hydrogel wrapped copper wire for fiber-based flexible all-solid-state asymmetric supercapacitors. J. Mater. Chem. A 3, 11215-11223 (2015).

51. J. Ren, W. Bai, G. Guan, Y. Zhang, and H. Peng: Flexible and weaveable capacitor wire based on a carbon nanocomposite fiber. Adv. Mater. 25, 5965-5970 (2013).

52. X. Chen, L. Qiu, J. Ren, G. Guan, H. Lin, Z. Zhang, P. Chen, Y. Wang, and H. Peng: Novel electric double-layer capacitor with a coaxial fiber structure. Adv. Mater. 25, 6436-6441 (2013).

53. W. Weng, Q. Sun, Y. Zhang, H. Lin, J. Ren, X. Lu, M. Wang, and H. Peng: Winding aligned carbon nanotube composite yarns into coaxial fiber full batteries with high performances. Nano Lett. 14, 3432-3438 (2014).

54. W. Liu, N. Liu, Y. Shi, Y. Chen, C. Yang, J. Tao, S. Wang, Y. Wang, J. Su, L. Li, and Y. Gao: A wire-shaped flexible asymmetric supercapacitor based on carbon fiber coated with a metal oxide and a polymer. J. Mater. Chem. A 3, 13461-13467 (2015).

55. Y. Fu, X. Cai, H. Wu, Z. Lv, S. Hou, M. Peng, X. Yu, and D. Zou: Fiber supercapacitors utilizing pen ink for flexible/wearable energy storage. Adv. Mater. 24, 5713-5718 (2012).

56. M. Shi, C. Yang, X. Song, L. Zhao, J. Liu, P. Zhang, and L. Gao: Integrated sustainable wind power harvesting and ultrahigh energy density wire-shaped supercapacitors based on vertically oriented nanosheet-array-coated carbon fibers. Adv. Sustainable Syst. 1, 1700044 (2017).

57. M.G. Mousa, S.M. Allam, and E.M. Rashad: Maximum power extraction under different vector-control schemes and gridsynchronization strategy of a wind-driven brushless doubly-fed reluctance generator. ISA Trans. 72, 287-297 (2018).

58. M. Sufyan, N.A. Rahim, M.M. Aman, C.K. Tan, and

S.R.S. Raihan: Sizing and applications of battery energy storage technologies in smart grid system: A review. J. Renewable Sustainable Energy 11, 014105 (2019).

59. J. Ren, Q. Hou, H. Chen, T. Liu, H. He, J. Wang, Q. Shao, M. Dong, S. Wu, N. Wang, J. Lin, Q. Luo, and Z. Guo:
Suppressing charge recombination and ultraviolet light degradation of perovskite solar cells using silicon oxide passivation. ChemElectroChem 6, 3167-3174 (2019).

60. B. Lin, Z. Lin, S. Chen, M. Yu, W. Li, Q. Gao, M. Dong, Q. Shao, S. Wu, T. Ding, and Z. Guo: Surface intercalated spherical $\mathrm{MoS}_{2 x} \mathrm{Se}_{2(1-x)}$ nanocatalysts for highly efficient and durable hydrogen evolution reactions. Dalton Trans. 48, 82798287 (2019)

61. Z. Lin, B. Lin, Z. Wang, S. Chen, C. Wang, M. Dong, Q. Gao, Q. Shao, T. Ding, H. Liu, S. Wu, and Z. Guo: Facile preparation of $1 \mathrm{~T} / 2 \mathrm{H}-\mathrm{Mo}\left(\mathrm{S}_{1-x} \mathrm{Se}_{x}\right)_{2}$ nanoparticles for boosting hydrogen evolution reaction. ChemCatChem 11, 2217-2222 (2019).

62. M. Liu, Z. Yang, H. Sun, C. Lai, X. Zhao, H. Peng, and T. Liu: A hybrid carbon aerogel with both aligned and interconnected pores as interlayer for high-performance lithium-sulfur batteries. Nano Res. 9, 3735-3746 (2016).

63. M. Liu, Q. Meng, Z. Yang, X. Zhao, and T. Liu: Ultra-long-term cycling stability of an integrated carbon-sulfur membrane with dual shuttle-inhibiting layers of graphene "nets" and a porous carbon skin. Chem. Commun. 54, 5090-5093 (2018).

64. R. Li, X. Zhu, Q. Fu, G. Liang, Y. Chen, L. Luo, M. Dong, Q. Shao, C. Lin, R. Wei, and Z. Guo: Nanosheet-based $\mathrm{Nb}_{12} \mathrm{O}_{29}$ hierarchical microspheres for enhanced lithium storage. Chem. Commun. 55, 2493-2496 (2019).

65. M. Idrees, S. Batool, J. Kong, Q. Zhuang, H. Liu, Q. Shao, N. Lu, Y. Feng, E.K. Wujcik, Q. Gao, T. Ding, R. Wei, and Z. Guo: Polyborosilazane derived ceramics-nitrogen sulfur dual doped graphene nanocomposite anode for enhanced lithium ion batteries. Electrochim. Acta 296, 925-937 (2019).

66. C. Wang, F. Lan, Z. He, X. Xie, Y. Zhao, H. Hou, L. Guo, V. Murugadoss, H. Liu, Q. Shao, Q. Gao, T. Ding, R. Wei, and Z. Guo: Iridium-based catalysts for solid polymer electrolyte electrocatalytic water splitting. ChemSusChem 12, 1576-1590 (2019). 